\title{
Educação popular em saúde do idoso: análise dos anais do ENEX com publicação no período de 2013 a $2017^{1}$
}

\author{
Klebson Felismino Bernardo \\ Graduação em Pedagogia pela UFPB. Mestrando em Educação pela UFPB \\ $\square$ klebsonbernardo987@hotmail.com \\ Arleciane Emilia de Azevêdo Borges \\ Graduação em Direito pelo UNIPÊ. Mestranda em Educação pela UFPB \\ Kathy Souza Xavier de Araújo \\ Graduação em Pedagogia pelo UNIGRAN. Mestranda em Educação pela UFPB
}

Recebido em 12 de agosto de 2020

Aceito em 2 de dezembro de 2021

\begin{abstract}
Resumo:
A Educação Popular em Saúde (EPS) do Idoso compreende-se como ações e saberes que contam com experiências e vivências no uso de práticas alternativas e/ou populares na saúde do idoso. Trata-se de um levantamento bibliográfico do que tem sido pesquisado a respeito da EPS do Idoso. Para tanto, foi realizada a coleta de dados, em outubro de 2019, através da observação indireta de artigos e resumos publicados nos Anais do Encontro Unificado de Ensino, Pesquisa e Extensão (ENEX) da Universidade Federal da Paraíba (UFPB), no período de 2013 a 2017. Os dados coletados foram transcritos em tabelas e apresentados por meio da abordagem descritiva, totalizando uma amostra de 13 trabalhos. Os idosos foram considerados de forma plena em todos os trabalhos avaliados. Conceitos como protagonismo, valorização do idoso, diálogo, respeito são defendidos por todos os autores. Ademais, as práticas educativas e a relação dos projetos de extensão da UFPB interagem com as comunidades de forma responsável e compromissada com o desenvolvimento dos sujeitos mediante o desempenho e a atuação dos coordenadores e extensionistas de cada projeto. Conclui-se que, embora diversos projetos de extensão da UFPB descrevam a interação com as comunidades de forma dialogada e compromissada, estudos envolvendo os idosos foram pouco explorados. Logo, sinaliza-se a relevância de discutir a construção de novos saberes, as experiências e o estabelecimento de diálogo entre universidade e comunidade a fim de fortalecer os vínculos e perpetuar os trabalhos científicos.

Palavras-chave: Saúde do Idoso, Atenção Integral ao Idoso, Cuidados Integrais de Saúde, Extensão.

\section{Popular education in health of the elderly: analysis of the annals of ENEX published between 2013 and 2017}

\begin{abstract}
:
Popular Health Education (EPS) for the elderly is understood as actions and knowledge that rely on experiences in the use of alternative and / or popular practices in the health of the elderly. It is a bibliographic survey of what has been researched about the EPS of the Elderly. To this end, data collection was carried out in October 2019, through indirect observation of articles and abstracts published in the Proceedings of the Unified Meeting of Teaching, Research and Extension (ENEX) of

\footnotetext{
1 Artigo fruto da participação discente durante o ano de 2019 no Projeto de Extensão intitulado "Educação Popular na Construção da Integralidade da Formação e do Cuidado em Saúde - EPI Saúde”, vinculado ao Departamento de Odontologia Restauradora do Centro de Ciências de Saúde da Universidade Federal da Paraíba
} 
the Federal University of Paraíba (UFPB), in the period of 2013 to 2017. The data collected were transcribed in tables and presented using the descriptive approach, totaling a sample of 13 works. The elderly were fully considered in all evaluated works. Concepts such as protagonism, valuing the elderly, dialogue, respect are defended by all authors. Furthermore, the educational practices and the relationship of the UFPB extension projects interact with the communities in a responsible and committed way with the development of the subjects through the performance and performance of the coordinators and extensionists of each project. It is concluded that, although several UFPB extension projects describe the interaction with the communities in a dialogical and committed way, studies involving the elderly were little explored. Therefore, the relevance of discussing the construction of new knowledge, experiences and the establishment of dialogue between the university and the community is highlighted in order to strengthen the bonds and perpetuate scientific work.

Keywords: Health of the Elderly, Comprehensive Care for the Elderly, Comprehensive Health Care, Extension.

\section{Educación popular en salud de las personas mayores: análisis de los anales de ENEX publicados entre 2013 y 2017}

\section{Resumen:}

La Educación de Salud Popular (EPS) para los ancianos se entiende como acciones y conocimientos que se basan en experiencias en el uso de prácticas alternativas y / o populares en la salud de los ancianos. Es una encuesta bibliográfica de lo que se ha investigado sobre el EPS de los ancianos. Con este fin, la recopilación de datos se llevó a cabo en octubre de 2019, a través de la observación indirecta de artículos y resúmenes publicados en las Actas de la Reunión Unificada de Docencia, Investigación y Extensión (ENEX) de la Universidad Federal de Paraíba (UFPB), en el período de 2013 a 2017. Los datos recopilados se transcribieron en tablas y se presentaron utilizando el enfoque descriptivo, totalizando una muestra de 13 obras. Los ancianos se consideraron plenamente en todos los trabajos evaluados. Conceptos como el protagonismo, la valoración de las personas mayores, el diálogo, el respeto son defendidos por todos los autores. Además, las prácticas educativas y la relación de los proyectos de extensión de UFPB interactúan con las comunidades de manera responsable y comprometida con el desarrollo de los temas a través del desempeño y el desempeño de los coordinadores y extensionistas de cada proyecto. Se concluye que, aunque varios proyectos de extensión de UFPB describen la interacción con las comunidades de una manera dialógica y comprometida, los estudios que involucraron a personas de edad avanzada fueron poco explorados. Por lo tanto, se destaca la importancia de discutir la construcción de nuevos conocimientos, experiencias y el establecimiento del diálogo entre la universidad y la comunidad para fortalecer los lazos y perpetuar el trabajo científico.

Palabras clave: Salud de los Ancianos, Atención Integral para Personas Mayores, Atención Integral de Salud, Extensión.

\section{INTRODUÇÃO}

O envelhecimento populacional implica um importante acontecimento mundial, que constitui no campo da saúde um desafio no que se refere a organizar práticas de saúde que considerem a complexidade do processo de envelhecimento. Para isso, se faz necessário proporcionar ações integrais de saúde que respondam às necessidades dessa população e colabore para sua qualidade de vida (CAMARANO; PASINATO, 2004). 
Em todo o mundo a dimensão de idosos desenvolve-se mais do que outras faixas etárias, sendo um desafio proporcionar o envelhecimento de forma ativa, através de políticas e programas que aumentem as possibilidades para saúde, segurança, autonomia e, consequentemente, bem-estar. A promoção do envelhecimento ativo e saudável faz parte de uma das diretrizes da Política Nacional de Saúde da Pessoa Idosa no Brasil em conformidade com a concepção atual da promoção da saúde (PEDROSA, 2014). No Brasil, o envelhecimento populacional foi influenciado por múltiplos fatores e resultados mostraram que a expectativa de vida ao nascer alcançou 74,1 anos em 2011 (IBGE, 2010).

A promoção da saúde procura operar uma concepção positiva de saúde que pressupõe a participação dos múltiplos atores sociais (crianças, jovens, adultos e etc.) e a aplicação de diversas estratégias, compreendendo assim a educação, a comunicação e a legislação. Faz-se necessário entender que a Educação em Saúde é uma área de práticas e saberes norteada por distintas perspectivas teórico-conceituais e políticas (RABELLO, 2010).

A Educação Popular em Saúde (EPS) pode ser entendida como um modo particular de reconhecer e encarar os problemas de saúde por meio do diálogo com as classes populares e do respeito às suas culturas, além do reconhecimento dos seus saberes como válidos e tendo como fundamento o corpo teórico da Educação Popular, orientada por Paulo Freire no Brasil. Vale destacar que a EPS tem sido reconhecida como referência para as práticas educativas no Sistema Único de Saúde (SUS), por sua consonância com o ideário de humanização, integralidade e participação popular (ALVES, 2017).

A EPS é orientada sob os pilares dos princípios teórico-metodológicos e das concepções pedagógicas de Paulo Freire. Esses princípios constituem a prática educativa e o trabalho social emancipatórios, intencionalmente orientados à promoção da autonomia das pessoas, à constituição da consciência crítica, como também à cidadania participativa e à superação das desigualdades sociais. As ações de promoção da saúde, fundamentadas na prática de educação em saúde, necessitam buscar a transformação da realidade, além de proporcionar o protagonismo dos sujeitos, de modo que reflitam sua realidade e sobre o que está a sua volta.

A EPS possibilita que o indivíduo reconheça-se enquanto sujeito de direitos e deveres, refletindo sobre sua realidade e, principalmente, tentando de forma consciente modificar 
essa realidade através de mecanismos críticos e reflexivos. No tocante a esse viés, pode-se inferir que, no movimento da promoção da saúde, é possível reconhecer a concepção da Educação em Saúde como processo político de constituição para a cidadania e ação renovadora sobre a realidade social (HOFFMANN; MAXIMO, 2019).

O objetivo do estudo consiste em levantamento bibliográfico acerca de EPS do Idoso a partir de artigos e resumos publicados nos Anais do Encontro Unificado de Ensino, Pesquisa e Extensão (ENEX) da Universidade Federal da Paraíba (UFPB) no período de 2013 a 2017.

\section{METODOLOGIA}

A pesquisa foi obtida por meio da observação indireta de artigos e resumos publicados nos Anais do ENEX da UFPB, no período de 2013 a 2017, com as palavras-chave: Saúde do Idoso; Atenção Integral ao Idoso; Cuidados Integrais de Saúde; Extensão.

O referido evento, realizado anualmente, constitui-se em um espaço para partilha das experiências vivenciadas nas atividades de extensão, contando com participantes extensionistas que atuam em ações regulamentadas pelos editais PROBEX, UFPB no seu município, FLUEX, PROTEC- ETS e pelas chamadas públicas (cursinhos). Toda a equipe de extensionistas dos projetos pode participar do ENEX; no entanto, discentes - na condição de bolsista ou voluntário - e coordenadores dos projetos de extensão devem publicar trabalhos oriundos das experiências acadêmicas, cabendo aos discentes a apresentação e aos docentes a submissão deles no SigEventos.

O estudo é de caráter exploratório e de abordagem quantitativa (MARCONI; LAKATOS, 2001; RICHARDSON, 1999). Utilizou-se como critério para inclusão na amostra: estudos desenvolvidos com ênfase na EPS do Idoso e trabalhos científicos nas modalidades de artigo e resumo. Foram estabelecidas como dimensões de análise as seguintes variáveis: (1) ano, (2) saúde do idoso, (3) promoção de hábitos alimentares saudáveis, (4) autonomia e (5) integralidade. 
A coleta de dados foi realizada em outubro de 2019, totalizando uma amostra de 13 trabalhos. Os dados coletados foram transcritos em tabelas e apresentados por meio da abordagem descritiva.

\section{RESULTADOS}

Dentre os artigos e os resumos publicados nos períodos de 2013 a 2017, descreve-se abaixo aqueles que se enquadram nos critérios metodológicos propostos pelo presente trabalho mediante a análise de variáveis sobre a EPS do Idoso (Tabela 1, página seguinte).

Ressalta-se que, dos 194 estudos publicados em 2013, apenas 2 artigos contemplam a propositura da pesquisa.

De acordo com o artigo Promoção da qualidade de vida do idoso na atenção primária à saúde, identifica-se que, através do Projeto de Extensão Comunitária "Formação Humanizada do Profissional de Saúde", há a promoção da vivência aos universitários dos cursos de graduação em área de saúde, juntamente com a Unidade de Saúde da Família (USF) Timbó II e a comunidade ali próxima, ao processo saúde-doença na realidade da atenção básica à saúde, desde o ano de 2007.

Para Cavalcanti Neto et al. (2013), com a ampliação da longevidade, as práticas voltadas para a saúde do idoso têm obtido destaque em todos os níveis da gestão pública de saúde. $\mathrm{Na}$ atenção primária à saúde, existem grandes possibilidades para a ampliação de ações programáticas direcionadas para a terceira idade. A experiência de uma equipe de extensionistas da UFPB em conjunto à USF e o desenvolvimento destas atividades tem se confirmando como coeficiente de transformação e de educação para a população.

No artigo analisado, nota-se como os autores exploram a variável "autonomia", pois, segundo Ramos (2003), a autonomia é um importante determinante da saúde do idoso, isto é, sua capacidade de fazer de forma autônoma as atividades da vida cotidiana, a exemplo: tomar banho, comer, se vestir, sem o auxílio de terceiros. 
Tabela 1 - Anais do ENEX de 2013 a 2017 em Educação Popular em Saúde do Idoso

\begin{tabular}{|c|c|c|c|}
\hline TÍTULO & AUTORES & ANO & $\begin{array}{l}\text { MODALIDADE } \\
\text { DA } \\
\text { PUBLICAÇÃO }\end{array}$ \\
\hline $\begin{array}{l}\text { Promoção da qualidade de vida do idoso } \\
\text { na atenção primária à saúde. }\end{array}$ & CAVALCANTI NETO, B. F. et al. & 2013 & Artigo \\
\hline $\begin{array}{l}\text { Práticas integrais de educação popular e } \\
\text { saúde comunitária: reflexões a partir da } \\
\text { extensão popular. }\end{array}$ & CARDOSO, E. L. S. et al. & 2013 & Artigo \\
\hline $\begin{array}{l}\text { A Educação em Saúde como estratégia de } \\
\text { promoção da qualidade de vida de idosos. }\end{array}$ & SILVA, C. R. et al. & 2014 & Resumo \\
\hline $\begin{array}{l}\text { Cuidado em saúde como estratégia para } \\
\text { promoção da qualidade de vida do idoso. }\end{array}$ & BOTELHO, B. O. et al. & 2014 & Resumo \\
\hline $\begin{array}{l}\text { Promovendo saúde: exercício físico } \\
\text { auxiliando no controle do índice de massa } \\
\text { corporal e relação cintura quadril em } \\
\text { grupo de mulheres e idosas no município } \\
\text { de Areia-PB. }\end{array}$ & $\begin{array}{l}\text { RIBEIRO, R. X.; OLIVEIRA, B. } \\
\text { S.; CANDIDO, M. L. A. }\end{array}$ & 2014 & Resumo \\
\hline 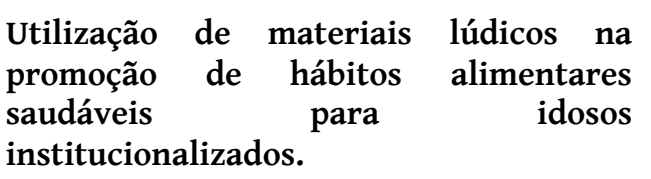 & SILVA, J. S. et al. & 2014 & Resumo \\
\hline $\begin{array}{l}\text { A educação em saúde como estratégia de } \\
\text { promoção da qualidade de vida de idosos. }\end{array}$ & BEZARRA, R. S. et al. & 2015 & Resumo \\
\hline $\begin{array}{l}\text { Programa educação popular em saúde do } \\
\text { trabalhador: uma experiência no grupo } \\
\text { operativo de idosos. }\end{array}$ & SANTOS, L. E. P. et al. & 2015 & Resumo \\
\hline $\begin{array}{l}\text { Relato de experiência: compreendendo a } \\
\text { história de vida de idosos } \\
\text { institucionalizados. }\end{array}$ & RIBEIRO, T. M. et al. & 2016 & Resumo \\
\hline $\begin{array}{l}\text { Programa mais saúde na comunidade: os } \\
\text { idosos e a saúde mental... contribuições } \\
\text { para viver mais e melhor!. }\end{array}$ & SILVA, A. M. M. et al. & 2016 & Resumo \\
\hline $\begin{array}{l}\text { Grupo de idosos "viver mais e melhor": } \\
\text { uma intervenção de extensão na saúde do } \\
\text { idoso orientado pela educação popular. }\end{array}$ & SILVA, A. M. M. et al. & 2017 & Resumo \\
\hline $\begin{array}{l}\text { Perfil dos participantes do projeto “Ações } \\
\text { Socioeducativas e Práticas de Promoção } \\
\text { da Saúde à Pessoa Idosa". }\end{array}$ & GREBOS, T. et al. & 2017 & Resumo \\
\hline $\begin{array}{l}\text { Rodas de conversa: um instrumento de } \\
\text { educação popular em saúde para pessoas } \\
\text { idosas. }\end{array}$ & BARROS, K. C. et al. & 2017 & Resumo \\
\hline
\end{tabular}

Fonte: Dados da Pesquisa, 2019. 
Corroborando com o exposto, é importante evidenciar que a Equipe da Unidade Saúde da Família Timbó II, Distrito Sanitário III do município de João Pessoa/PB, proporciona atenção primária à saúde para cerca de 760 famílias. Esta comunidade está situada na zona sul da capital paraibana e compreende área de risco social relevante. No que se refere à população de idosos adstrita, abrange cerca de 2.800 usuários (SANTOS, 2013).

Nas análises realizadas, o artigo intitulado Práticas integrais de educação popular e saúde comunitária: reflexões a partir da extensão popular traz a importância das práticas de extensão popular que utilizam a metodologia da Educação Popular, visando à constituição de ações geradoras de autonomia, em especial nos setores menos desfavorecidos da sociedade.

Nesse sentido, o Projeto "Práticas Integrais de Educação Popular e Saúde Comunitária", apresentado no artigo supracitado, explicita as ações e as reflexões com o objetivo de propiciar, na comunidade, a autonomia e a constituição de um olhar diferenciado e crítico no que lhe concerne a sua própria realidade. Infere-se que os artigos publicados no ENEX de 2013 destacam a variável "autonomia”.

As iniciativas desse projeto de extensão da UFPB “priorizam o exercício do diálogo e da escuta, permitindo o desvelamento de caminhos, saberes e práticas, em que o saber do outro é valorizado e, suas potencialidades, evidenciadas" (CARDOSO, et al., 2013, p. 2). É importante relatar que essas ações são desenvolvidas nas comunidades Jardim Itabaiana, Boa Esperança e Pedra Branca, situadas no bairro do Cristo Redentor, em João Pessoa/PB, principalmente em espaços como a USF Vila Saúde e Associação dos Amigos e Moradores da Boa Esperança.

Quanto ao ano de 2014, a pesquisa identifica que em um total de 585 trabalhos com descritor saúde, apenas 4 referem-se à EPS do Idoso.

O resumo A educação em saúde como estratégia de promoção da qualidade de vida de idosos traz a importância de se construir uma consciência crítica a cerca dos hábitos alimentares saudáveis. Além do mais, salienta e desenvolve por meio de um projeto de Educação em Saúde ações com a USF Santa Clara, localizada no bairro do Castelo Branco, no município de João Pessoa/PB, trabalhando com um grupo composto por aproximadamente 35 participantes. 
O resumo traz também a necessidade do diálogo para a construção de saberes e experiências de forma mútua, como também o exercício da autonomia dos idosos e a sua integralidade. Questões como prevenção de agravos à saúde - combate ao alcoolismo, ao tabagismo e a automedicação - e prevenção de quedas são presentes nas ações propostas na USF.

Já o resumo Cuidado em saúde como estratégia para promoção da qualidade de vida do idoso, de autoria dos extensionistas do Programa Práticas Integrais de Promoção da Saúde e Nutrição na Atenção Básica (PINAB), além da integralidade da pessoa idosa, atua na busca da autonomia, dos hábitos saudáveis e da saúde do idoso. A emancipação humana dos sujeitos também é um dos objetivos descritos nesse trabalho.

O resumo Promovendo saúde: exercício físico auxiliando no controle do índice de massa corporal e relação cintura quadril em grupo de mulheres e idosas no município de Areia-PB destaca a necessidade de se discutir acerca do sedentarismo dos idosos junto com as alterações fisiológicas, sendo observados com o passar dos anos e isso implica um dos principais fatores de risco à saúde, por causa do acúmulo de adiposidade corporal e redução do rendimento cardiorrespiratório.

Quanto ao resumo Utilização de materiais lúdicos na promoção de hábitos alimentares saudáveis para idosos institucionalizados, de autoria dos extensionistas do Projeto de Extensão Melhor Idade com Alimentação e Nutrição de Qualidade, pode-se perceber que o projeto é voltado para a melhoria dos hábitos alimentares dos idosos institucionalizados, no município de João Pessoa/PB.

O resumo comenta a importância de hábitos alimentares saudáveis e defende a necessidade do protagonismo da pessoa idosa em sua realidade, porém com a consciência crítica e reflexiva sobre como atuar socialmente e sobre o que ingerir em prol de sua saúde.

No ano de 2015, no campo da saúde com os descritores listados, foram publicados 571 resumos nos Anais do ENEX, porém apenas 2 resumos se propõem a abordar o idoso sob o olhar da EPS.

O resumo A educação em saúde como estratégia de promoção da qualidade de vida de idosos discorre a respeito do cuidado da pessoa idosa e explicita sobre a importância de se contribuir para a valorização da vida, do autocuidado, do crescimento pessoal e busca ativa da saúde no 
dia a dia através da aprendizagem participativa. Identifica-se também que a saúde do idoso precisa ser discutida, colocando os sujeitos idosos de forma ativa e participativa na própria vida e na sociedade, visto que esses sujeitos são partícipes nas tomadas de decisão socialmente.

O resumo Programa educação popular em saúde do trabalhador: uma experiência no grupo operativo de idosos aborda a melhoria da qualidade de vida da pessoa idosa, possibilitando a sua inserção na sociedade com autonomia necessária para a sua sobrevivência. Tal trabalho é fruto da interação do Programa de Educação Popular em Saúde do Trabalhador (PEPST) com idosos da comunidade do Grotão, no município de João Pessoa/PB. Nesse sentido, evidenciase que o resumo defende a ideia da melhoria de vida dos idosos, do seu protagonismo e da sua integralidade.

Os dados de 2016 demonstram que 406 dos resumos discutem a saúde como um todo e que o interesse pelo estudo da EPS do Idoso continua sendo pouco abordado.

Com referência ao resumo Relato de experiência: compreendendo a história de vida de idosos institucionalizados, a educação em saúde estabelece um processo que objetiva contribuir com a melhoria da qualidade de vida dos sujeitos através do desenvolvimento de ações de promoção a saúde, procurando, ainda, incitar um senso crítico sobre os problemas presentes na comunidade e as intervenções imprescindíveis para resolução dos mesmos.

Nesse resumo, observa-se que o Projeto "Saúde do idoso: vivência do processo saúdedoença dos residentes da Vila Vicentina Júlia Freire" possui o papel de contribuir por meio do diálogo com os idosos, resgatando a autoestima, além de pautar sobre informações particulares dos mais diferentes temas, a saber: famílias, amizades, saúde, sexualidade e convivência na instituição (RIBEIRO et al., 2016).

O resumo Programa mais saúde na comunidade: os idosos e a saúde mental... contribuições para viver mais e melhor!, cuja autoria é dos extensionistas do Grupo de Idosos do Programa Mais Saúde na Comunidade da UFPB, elenca questões como: condições socioeconômicas, ambientais, psíquicas e emocionais; hábitos de vida, aspectos culturais; entre outras.

O encontro com o grupo de idosos é realizado semanalmente aos sábados, na Escola Municipal de Ensino Fundamental Tharcilla Barbosa da Franca, no bairro do Grotão, em João 
Pessoa/PB. De acordo com a análise do resumo, esse grupo de idosos promove a valorização dos idosos e dos alunos como sujeitos independentes, construindo processos compartilhados e participativos e, através dessa interação, tem-se permitido o estímulo ao cuidado integral em saúde mental desses sujeitos. As variáveis que foram pontuadas nesse trabalho são autonomia, saúde do idoso e integralidade.

O período de 2017 foi marcado por um quantitativo de 3 resumos que remontam a EPS do Idoso, sendo selecionados dentre 315 resumos.

Sobre o resumo Grupo de idosos "viver mais e melhor": uma intervenção de extensão na saúde do idoso orientado pela educação popular, sua construção é fruto da experiência do Programa "Mais Saúde na Comunidade" da UFPB. A localidade onde está situado o grupo é o bairro do Grotão, zona sul da periferia do município de João Pessoa/PB.

O grupo tem como finalidade trocar informações a respeito de saberes e práticas sobre envelhecimento saudável, em que o fio condutor é a EPS. Para além, o trabalho pontua "princípio do diálogo horizontal ("troca de saberes"), problematização, amorosidade, construção compartilhada e participativa de conhecimentos, autonomia e protagonismo dos sujeitos" (SILVA et al., 2017, p. 741).

O resumo Perfil dos participantes do projeto "Ações Socioeducativas e Práticas de Promoção da Saúde à Pessoa Idosa" descreve o perfil sociodemográfico dos idosos participantes do projeto de extensão denominado "Aç̃oes socioeducativas e práticas de promoção da saúde à pessoa idosa". Questões como protagonismo, valorização desses sujeitos, consciência crítica e autonomia são discutidos nesse trabalho.

Referindo-se ao resumo intitulado Rodas de conversa: um instrumento de educação popular em saúde para pessoas idosas, discute-se o incentivo à construção de uma consciência crítica $\mathrm{e}$ à cooperação para o empoderamento da pessoa idosa através de trocas de conhecimentos e experiências entre todos os participantes. Observa-se que a escuta é um dos conceitos defendidos pelos autores, abrindo espaços para o diálogo e para a construção dos sujeitos, uma vez que praticar a escuta implica o reconhecimento da humildade dos indivíduos e a valorização dos conhecimentos populares e científicos como saberes que se complementam. 


\section{DISCUSSÃO}

Enfatiza-se que as publicações nos Anais do ENEX com a abordagem da EPS do Idoso, no período de 2013 a 2017, ainda são escassas, ainda que projetos de extensão da UFPB tenham atuação significativa nas comunidades do município de João Pessoa/PB.

As variáveis que mais se destacaram nos artigos e resumos foram: autonomia, integralidade e promoção de hábitos alimentares saudáveis. Através das análises das publicações concernentes à EPS do Idoso, constata-se que há a valorização do idoso e de seu protagonismo na sociedade.

Os idosos foram considerados de forma plena em todos os trabalhos avaliados. Conceitos como protagonismo, valorização do idoso, diálogo, respeito são defendidos por todos os autores. Ademais, as práticas educativas e a relação dos projetos de extensão da UFPB interagem com as comunidades de forma responsável e compromissada com o desenvolvimento dos sujeitos mediante o desempenho e a atuação dos coordenadores e extensionistas de cada projeto.

O município de João Pessoa/PB é o local em que todos esses trabalhos se desenvolveram em parceria com as comunidades do Timbó I, Timbó II, Grotão, Valentina de Figueiredo, Pedra Branca, dentre outras descritas na pesquisa.

A prática da educação popular em saúde permite a aproximação do extensionista com a perspectiva integral do cuidado na conjuntura familiar, levando em consideração também os aspectos econômicos, sociais e políticos que abrangem o processo saúde-doença do sujeito, família e comunidade (BANDEIRA; LOPES, 2018).

No tocante à educação em saúde, frisa-se que este é um dos principais dispositivos para possibilitar e concretizar a promoção da saúde, já que amplia o desenvolvimento da responsabilidade individual e coletiva para a prevenção de agravos (SANTOS et al., 2018). Conforme essa realidade, imprescindível realçar que se estima que em 2025 o Brasil venha a se tornar o sexto maior país em quantitativo de idosos do mundo. Portanto, com base na importância e na contemporaneidade da temática, não seria exagero dizer que os estudos referentes sobre as pessoas longevas é um campo ainda novo e desafiador (BRAGA; GALLEGUILLOS, 2018). 
Assim, faz-se fundamental a ampliação do conhecimento referente ao idoso, pois as discussões ressaltam a importância de se ter um olhar humanizado para essa população quanto à pertinência de seu espaço, subjetividades, saberes e experiências.

\section{CONSIDERAÇÕES FINAIS}

Diante do exposto, houve uma diminuição de produções referentes à saúde nas publicações nos Anais do ENEX no lapso temporal estudado, notadamente poucos trabalhos acerca da EPS do Idoso, considerando diversos projetos de extensão, que abordam essa temática, existentes na UFPB.

A pesquisa percebe que os estudos analisados foram importantes para a visibilidade dos projetos extensionistas, evidenciando a população idosa como sujeitos sociais e históricos. Por isso, faz-se necessário entender que a EPS do Idoso deve ser cada vez mais discutida e que a comunidade acadêmica precisa potencializar o olhar sobre essa perspectiva, uma vez que os idosos fazem parte da sociedade e, assim, de suas decisões pertinentes.

Logo, sinaliza-se a relevância de discutir a construção de novos saberes, as experiências e o estabelecimento de diálogo entre universidade e comunidade a fim de fortalecer os vínculos e perpetuar os trabalhos científicos.

\section{REFERÊNCIAS}

ALVES, M. R. Educação Popular em Saúde: um referencial de autonomia e transformação. Dissertação (Mestrado em Educação) - Universidade Federal dos Vales do Jequitinhonha e Mucuri, Diamantina/MG, 2017.

BANDEIRA, H. M. M.; LOPES, M. S. L. Encontro com a Didática: tecendo fios com a educação e a saúde. Curitiba: Appris Editora e Livraria Eireli-ME, 2018.

BARROS, K. C.; LIMA, R. S.; SÁ, R. B. C. P.; FONTES, P. C.; AMARAL, A. K. F. J. Rodas de conversa: um instrumento de educação popular em saúde para pessoas idosas. 2017. Anais do ENEX. João Pessoa: UFPB, 2017.

BEZARRA, R. S.; MONTEIRO, I. X.; FARIAS, R.; MONTEIRO, E. A.; PONTES, M. L. F. A educação em saúde como estratégia de promoção da qualidade de vida de idosos. 2015. Anais do ENEX. João Pessoa: UFPB, 2015. 
BOTELHO, B. O.; NASCIMENTO, E. N. F.; LIMA, G. N. S.; CRUZ, P. J. S. C.; BARRETO, R. G.; VENCESLAU, S. C. A. P. Cuidado em saúde como estratégia para promoção da qualidade de vida do idoso. 2014. Anais do ENEX. João Pessoa: UFPB, 2014.

BRAGA, C.; GALLEGUILlOS, T. G. B. Saúde do adulto e do idoso. São Paulo: Saraiva Educação S.A., 2018.

CAMARANO, A. A.; PASINATO, M. T. O envelhecimento populacional na agenda das políticas públicas. In: CAMARANO, A. A. (Org.). Os novos idosos brasileiros: muito além dos 60? Rio de Janeiro: IPEA, 2004.

CARDOSO, E. L. S.; ALVES, A. S.; CARNEIRO, D. G. B.; CRUZ, P. J. S. C.; VASCONCELOS, M. O. D. Práticas integrais de educação popular e saúde comunitária: reflexões a partir da extensão popular. 2013. Anais do ENEX. João Pessoa: UFPB, 2013.

CAVAlCANTI NETO, B. F.; PRIMO, G. N.; FARIAS, R. L. G. P.; AGUAR, F. B.; HIRSCH-MONTEIRO, C. Promoção da qualidade de vida do idoso na atenção primária à saúde. 2013. Anais do ENEX. João Pessoa: UFPB, 2013.

GREBOS, T.; AMARAL, A. K. F. J.; PONTES, E. S.; FONSECA, I. C. D. A.; COSTA, T. D. C. Perfil dos participantes do projeto “Ações Socioeducativas e Práticas de Promoção da Saúde à Pessoa Idosa". 2017. Anais do ENEX. João Pessoa: UFPB, 2017.

HOFFMANN, J.; MAXIMO, C. E. A Educação Popular em Saúde como dispositivo transformador das práticas de produção da saúde no município de Itajaí-SC. Revista Pesquisas e Práticas Psicossociais, São João Del-Rei, v. 14, n. 1, p. 1-14, 2019.

INSTITUTO BRASILEIRO DE GEOGRAFIA E ESTATÍSTICA (IBGE). Números gerais do censo. 2010. Disponível em: <https://censo2010.ibge.gov.br/index.php>. Acesso em: 24 jun. 2020.

MARCONI, M. A.; LAKATOS, E. M. Metodologia científica. São Paulo: Editora Atlas, 2001.

PEDROSA, W. C. Envelhecimento ativo: um desafio para a equipe multidisciplinar e para as pessoas idosas frequentadoras da Unidade de Atenção ao Idoso (UAI), no município de Uberaba-MG. Dissertação (Mestrado em Serviço Social) - Universidade Estadual Paulista "Júlio de Mesquita Filho", Franca/SP, 2014.

RABELLO, L. S. Promoção da saúde: a construção social de um conceito em perspectiva comparada. Rio de Janeiro: Editora Fiocruz, 2010.

RAMOS, L. R. Fatores determinantes do envelhecimento saudável em idosos residentes em centros urbanos: Projeto Epidoso, São Paulo. Cadernos de Saúde Pública, Rio de Janeiro, v. 19, n. 3, p. 793-797, jun., 2003.

RIBEIRO, R. X.; OLIVEIRA, B. S.; CANDIDO, M. L. A. Promovendo saúde: exercício físico auxiliando no controle do índice de massa corporal e relação cintura quadril em grupo de mulheres e idosas no município de Areia-PB. 2014. Anais do ENEX. João Pessoa: UFPB, 2014.

RIBEIRO, T. M.; SERAFIM, C. A. L.; MOURA, W. S. M.; LEITE, T. A.; BATISTA, L. M. Relato de experiência: compreendendo a história de vida de idosos institucionalizados. 2016. Anais do ENEX. João Pessoa: UFPB, 2016.

RICHARDSON, R. J. Pesquisa social: métodos e técnicas. São Paulo: Atlas, 1999.

SANTOS, M. E. D. Relato de vivência de uma médica de saúde da família e comunidade. João Pessoa: Idea, 2013. 202p.

SANTOS, L. E. P.; SILVA, A. M. M.; CAMPOS, J. M. A.; RAMALHO, E. L. R.; LACERDA, D. A. L. Programa educação popular em saúde do trabalhador: uma experiência no grupo operativo de idosos. 2015. Anais do ENEX. João Pessoa: UFPB, 2015.

SANTOS, V. P.; LIMA, W. R.; ROSA, R. S.; BARROS, I. M. C.; BOERY, R. N. S. O.; CIOSAK, S. I. Perfil de saúde de idosos muito velhos em vulnerabilidade social na comunidade. Revista Cuidarte, Colômbia, v. 9, n. 3, p. 2322-2337, 2018. 
SILVA, C. R.; JESUS, D. R. R.; MONTEIRO, E. A.; ARAÚJO, E. G. S.; PONTES, M. L. F.; SILVA JUNIOR, S. V. A Educação em Saúde como estratégia de promoção da qualidade de vida de idosos. 2014. Anais do ENEX. João Pessoa: UFPB, 2014.

SILVA, J. S.; SILVA, L. R. G.; MAMEDE, L. C. G. P.; LINS, P. R. M.; AQUINO, J. S. Utilização de materiais lúdicos na promoção de hábitos alimentares saudáveis para idosos institucionalizados. 2014. Anais do ENEX. João Pessoa: UFPB, 2014.

SILVA, A. M. M.; ARAÚJO, E. K. G.; SILVA, N. F.; MARTHA, T. A.; LACERDA, D. A. L. Programa mais saúde na comunidade: os idosos e a saúde mental... contribuições para viver mais e melhor! 2016. Anais do ENEX. João Pessoa: UFPB, 2016.

SILVA, A. M. M.; ASSIS, M. M. G.; ARAUJO, N. E.; LACERDA, D. A. L. Grupo de idosos "viver mais e melhor": uma intervenção de extensão na saúde do idoso orientado pela educação popular. 2017. Anais do ENEX. João Pessoa: UFPB, 2017.

\section{$(\mathrm{cc}) \mathrm{Br}$}

Este trabalho está licenciado com uma Licença Creative Commons - Atribuição 4.0 Internacional. 